\title{
Numerical Approach for Solving Fractional Pantograph Equation
}

\author{
Ayse Anapali \\ Department of Mathematics \\ Mugla Sıtkı Koçman University \\ Turkey
}

\author{
Yalçın Öztürk \\ Ula Ali Koçman Vocational \\ School \\ Turkey
}

\author{
Mustafa Gülsu \\ Department of Mathematics \\ Mugla Sıtkı Koçman University \\ Turkey
}

\begin{abstract}
In this article, we have investigate a Taylor collocation method, which is based on collocation method for solving fractional pantograph equation. This method is based on first taking the truncated fractional Taylor expansions of the solution function in the mathematical model and then substituting their matrix forms into the equation. Using the collocation points, we have the system of nonlinear algebraic equation. Then, we solve the system of linear algebraic equation using Maple 14 and we obtain the coefficients of Taylor expansion. In addition illustrative example is presented to demonstrate the effectiveness of the proposed method.
\end{abstract}

\section{Keywords}

Fractional pantograph equation; pantograph equation; fractional differential equation; mathematical model; collocation method; approximate solution

\section{INTRODUCTION}

Nowadays notable contributions have been made theory and applications of the fractional differential equations(FDEs). Many problem can be modelling with the help of the FDEs in many areas such as seismic analysis, viscous damping, viscoelastic materials and polymer physics[1-3].

The pantograph equation is a kind of delay differential equation which is used different fields of pure and applied mathematics such as number theory, dynamical systems, probability, quantum mechanics and electrodynamics. Pantograph equation was studied by many authors and solved several numerical methods. The most important them are collocation method [4], spline method [5], Runga-Kutta method [6], Adomian decomposition method [7], homotopy perturbation method [8] etc. There are several pantograph equation kind in literature. One of them is multi Pantograph equation and this equation is defined by $[6,9,10]$

$u^{\prime}(t)=a u(t)+\sum_{i=1}^{m} \mu_{i}(t) u\left(\lambda_{i} t\right)+f(t), t \geq 0$

Another form of of pantograph equation is generalized nonlinear multi pantograph equation of the form [11]

$u^{\prime}(t)=f\left(t, u(t), u\left(\lambda_{1} t\right), \cdots, u\left(\lambda_{m} t\right)\right)$

The general pantograph equation is defined by

$y^{\prime}(x)=a y(x)+b y(q x), 0 \leq x \leq x_{f}, y(0)=y_{0}$

where $0<q<1[12]$.

Pantograph equation has very importance in applied science because of this reason fractional model of pantograph equation has been studied many researchers. Most fractional differential equations do not have exact analytic solutions, so approximation and numerical techniques must be used. For example Adomian decomposition method, the homotopyperturbation method, the variational iteration method and the homotopy analysis method [13-19]. In this paper we use the collocation method for solving fractional Pantograph differential equation

$D_{*}^{i \alpha} y(x)=f(x)+\sum_{r=0}^{m} p_{r}(x) y\left(q_{r} x\right)$

$a \leq x \leq b, n-1 \leq m \alpha<n$

under the initial conditions

$D_{*}^{i} y(c)=\lambda_{i}, a \leq c \leq b, i=0,1, \ldots, n-1$

where $0<q_{r}<1$ and $\lambda_{i}$ is appropriate constant.

We investigate the approximate solution of Eq.(1) under the conditions Eq.(2) with the fractional Taylor series as

$$
\begin{aligned}
& D_{a}^{k \alpha} y(x) \in C(a, b], \\
& y_{N}(x)=\sum_{i=0}^{N} \frac{(x-a)^{i \alpha}}{\Gamma(i \alpha+1)}\left(D_{a}^{i \alpha} y\right)(a)
\end{aligned}
$$

where $0<\alpha \leq 1$. In recently, collocation method has become very useful technique for solving differential equations [2027]. This method transform each part of differential equation into matrix form and using the collocation points

$x_{i}=\frac{i}{N}, i=0,1, \ldots, N$

we get the linear algebraic equation. Solving this equation, we obtained the coefficients of Taylor polynomial, the

approximate solutions for various $N$. All computations are performed on the computer algebraic system Maple14 in this paper.

\section{BASIC DEFINITIONS}

In this section, we first give some basic definitions and then present properties of fractional calculus $[2,26]$.

Definition 2.1 A real function $f(x), x>0$, is said to be in space $C_{\mu}, \mu \in R$ if there exist a real number $p(>\mu)$, such that $f(x)=x^{p} f_{1}(x)$, where $f_{1}(x) \in[0, \infty)$, and it is said to be in the space $C_{\mu}^{m}$ iff $f^{(m)} \in C_{\mu}, m \in N$.

Definition 2.2 The Riemann-Liouville fractional derivative of order $\alpha$ with respect to the variable $t$ and with the starting point at $t=a$ is 
${ }_{a} D_{t}^{\alpha} f(t)=\left(\frac{d}{d t}\right)^{m+1} \int_{a}^{t}(t-\tau)^{m-\alpha} f(\tau) d \tau$,

$(m \leq \alpha<m+1)$.

Definition 2.3 The Riemann-Liouville fractional integral of order $\alpha$ is

${ }_{a} D_{t}^{-\alpha} f(t)=\frac{1}{\Gamma(\alpha)} \int_{a}^{t}(t-\tau)^{\alpha-1} f(\tau) d \tau, p>0$

Definition 2.4 The fractional derivative of $f(x)$ by means of Caputo sense is defined as

$D^{\alpha} f(t)=\frac{1}{\Gamma(n-\alpha)} \int_{0}^{t}(t-\tau)^{n-\alpha-1} f^{(n)}(\tau) d \tau$

for $n-1<\alpha \leq n, n \in \mathrm{N}, t>0, f \in C_{-1}^{n}$.

Definition 2.5 Riemann -Liouville fractional derivative ${ }_{a} D_{t}^{\alpha} f(t)$ of the power function $f(t)=(t-a)^{v}$, where v is areal number is

$\left.{ }_{a} D_{t}^{\alpha}(\mathrm{t}-\mathrm{a})^{v}=\frac{\Gamma(v+1)}{\Gamma(v-\alpha+1)}\right)(\mathrm{t}-\mathrm{a})^{\mathrm{v}-\alpha}$

Some properties of fractional derivative and fractional integral are listed below: [2,27]

1. $D^{\alpha}(\lambda f(t)+\mu f(t))=\lambda D^{\alpha} f(t)+\mu D^{\alpha} f(t), \lambda, \mu$ are constants.

2. ${ }_{a} D_{t}^{\alpha}\left({ }_{a} D_{t}^{-\alpha} f(t)\right)=f(t)$

3. ${ }_{a} D_{t}^{\alpha}\left({ }_{a} D_{t}^{\beta} f(t)\right)={ }_{a} D_{t}^{\alpha-\beta} f(t)$

4. $D_{*}^{\alpha} C=0$ for any constant $C$

Theorem 1. (Generalized Taylor Formula) [26] Suppose that $D_{a}^{k \alpha} f(x) \in C(a, b] \quad$ for $\quad k=0,1, \ldots, n+1 \quad$ where $0<\alpha \leq 1$, then we have

$f(x)=\sum_{i=0}^{n} \frac{(x-a)^{i \alpha}}{\Gamma(i \alpha+1)}\left(D_{a}^{i \alpha} f\right)(a)+\frac{\left(D_{a}^{(n+1)} f\right)(\xi)}{\Gamma((n+1) \alpha+1)}(x-a)^{(n+1) \alpha}$

with $a \leq \xi \leq x, \forall x \in(a, b]$, where

$D_{a}^{n \alpha}=D_{a}^{\alpha} \cdot D_{a}^{\alpha} \cdot D_{a}^{\alpha} \ldots . D_{a}^{\alpha} \quad(n$ times $)$.

\section{FUNDAMENTAL RELATIONS}

In this section, we consider the fractional Pantograph differential equations (1). We use the Taylor matrix method [20-27] to find the truncated fractional Taylor series expansions of each term in expression at $x=c$ and their matrix representations for solving $\alpha$-th order linear fractional part.

We first consider the solution $y(x)$ of Eq. (1) defined by a truncated fractional Taylor series (3). Then, we have the matrix form of the solution $y(x)$ as $\lfloor y(x)\rfloor=\mathbf{X M}_{0} \mathbf{A}$

where

$\mathbf{X}=\left[\begin{array}{lllll}1 & (x-c)^{\alpha} & (x-c)^{2 \alpha} & \cdots & (x-c)^{N \alpha}\end{array}\right]$

$\mathbf{M}_{0}=\left[\begin{array}{ccccc}\frac{1}{\Gamma(1)} & 0 & 0 & \cdots & 0 \\ 0 & \frac{1}{\Gamma(\alpha+1)} & 0 & \cdots & 0 \\ 0 & 0 & \frac{1}{\Gamma(2 \alpha+1)} & \cdots & 0 \\ \vdots & \vdots & \vdots & \ddots & \vdots \\ 0 & 0 & 0 & \cdots & \frac{1}{\Gamma(N \alpha+1)}\end{array}\right]$

$\mathbf{A}=\left[\begin{array}{c}D_{*}^{0 \alpha} y(c) \\ D_{*}^{1 \alpha} y(c) \\ D_{*}^{2 \alpha} y(c) \\ \vdots \\ D_{*}^{N \alpha} y(c)\end{array}\right]$

Similarly, the matrix representation of the function $D_{*}^{\alpha} y(x)$ become

$D_{*}^{\alpha} y(x)=D_{*}^{\alpha} \mathbf{X} \mathbf{M}_{0} \mathbf{A}$

where, we compute the $D_{*}^{\alpha} \mathbf{X}$, then

$$
\begin{aligned}
& D_{*}^{\alpha} \mathbf{X}=\left[\begin{array}{lllll}
D_{*}^{\alpha} 1 & D_{*}^{\alpha}(x-c)^{\alpha} & D_{*}^{\alpha}(x-c)^{2 \alpha} & \cdots & D_{*}^{\alpha}(x-c)^{N \alpha}
\end{array}\right]
\end{aligned}
$$

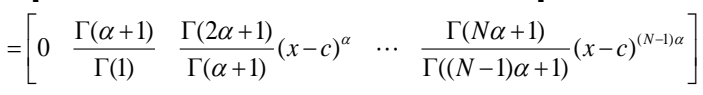

$$
\begin{aligned}
& =\mathbf{X} \mathbf{M}_{1}
\end{aligned}
$$

where

$\mathbf{M}_{1}=\left[\begin{array}{ccccc}0 & \frac{\Gamma(\alpha+1)}{\Gamma(1)} & 0 & \cdots & 0 \\ 0 & 0 & \frac{\Gamma(2 \alpha+1)}{\Gamma(\alpha+1)} & \cdots & 0 \\ \vdots & \vdots & \vdots & \ddots & \vdots \\ 0 & 0 & 0 & \cdots & \frac{\Gamma(N \alpha+1)}{\Gamma((N-1) \alpha+1)} \\ 0 & 0 & 0 & \cdots & 0\end{array}\right]$

then,

$D_{*}^{\alpha} y(x)=\mathbf{X} \mathbf{M}_{1} \mathbf{M}_{0} \mathbf{A}$

and

$$
\begin{aligned}
D_{*}^{2 \alpha} \mathbf{X} & =\left[\begin{array}{lllll}
D_{*}^{2 \alpha} 1 & D_{*}^{2 \alpha}(x-c)^{\alpha} & D_{*}^{2 \alpha}(x-c)^{2 \alpha} & \cdots & D_{*}^{2 \alpha}(x-c)^{N \alpha}
\end{array}\right] \\
& =\left[\begin{array}{lllll}
0 & 0 & \frac{\Gamma(2 \alpha+1)}{\Gamma(1)}(x-c)^{\alpha} & \cdots & \frac{\Gamma(N \alpha+1)}{\Gamma((N-2) \alpha+1)}(x-c)^{(N-2) \alpha}
\end{array}\right] \\
& =\mathbf{X M}_{2}
\end{aligned}
$$

where

$$
\mathbf{M}_{2}=\left[\begin{array}{ccccc}
0 & 0 & \frac{\Gamma(2 \alpha+1)}{\Gamma(1)} & \cdots & 0 \\
0 & 0 & 0 & \cdots & 0 \\
\vdots & \vdots & \vdots & \ddots & \vdots \\
0 & 0 & 0 & \cdots & \frac{\Gamma(N \alpha+1)}{\Gamma((N-2) \alpha+1)} \\
0 & 0 & 0 & \cdots & 0
\end{array}\right]
$$


Then, so the matrix representation of fractional differential part of equation can be written by

$D_{*}^{2 \alpha} y(t)=\mathbf{X} \mathbf{M}_{2} \mathbf{M}_{0} \mathbf{A}$

In similar way for any $i$, it can be written by

$D_{*}^{i \alpha} y(x)=\mathbf{X} \mathbf{M}_{i} \mathbf{M}_{0} \mathbf{A}$

where

$M_{i}=\left[\begin{array}{ccccccc}0 & 0 & \ldots & \frac{1}{\Gamma(1)} & 0 & \ldots & 0 \\ 0 & 0 & \ldots & 0 & \frac{1}{\Gamma(\alpha+1)} & \ldots & 0 \\ \vdots & \vdots & \ddots & \vdots & \vdots & \ddots & \vdots \\ 0 & 0 & \ldots & 0 & 0 & \ldots & \frac{1}{\Gamma((N-i) \alpha+1)} \\ 0 & 0 & \ldots & 0 & 0 & \ldots & 0 \\ \vdots & \vdots & \ddots & \vdots & \vdots & \ddots & \vdots \\ 0 & 0 & \ldots & 0 & 0 & \ldots & 0\end{array}\right]$

Finally, we obtained the matrix representation of the condition in given Eq.(2) as $i=0,1, \ldots, m-1$

$\mathbf{U}_{i}=\mathbf{X}(c) \mathbf{M}_{k}=\left[\begin{array}{lllll}u_{i 0} & u_{i 1} & u_{i 2} & \cdots & u_{i N}\end{array}\right]=\left[\lambda_{i}\right]$

\section{METHOD OF SOLUTIONS}

Using the collocation points in Eq.(1), we obtain

$$
\left(\mathbf{X M}_{i} \mathbf{M}_{0}-\sum_{r=0}^{m} \mathbf{P}_{\mathbf{r}} \mathbf{X} \mathbf{B}_{\mathbf{q}_{\mathbf{r}}, \mathbf{c}}\left(x_{i}\right) \mathbf{M}_{\mathbf{0}}\right) \mathbf{A}=F\left(x_{i}\right)
$$

and the matrix representation of fractional pantograph equation as

$$
\left(\mathbf{X M}_{i} \mathbf{M}_{0}-\sum_{r=0}^{m} \mathbf{P}_{\mathbf{r}} \mathbf{X} \mathbf{B}_{\mathbf{q}_{\mathbf{r}}, \mathbf{c}} \mathbf{M}_{\mathbf{0}}\right) \mathbf{A}=\mathbf{F}
$$

where

$$
\mathbf{X}=\left[\begin{array}{ccccc}
1 & \left(x_{0}-c\right)^{\alpha} & \left(x_{0}-c\right)^{2 \alpha} & \cdots & \left(x_{0}-c\right)^{N \alpha} \\
1 & \left(x_{1}-c\right)^{\alpha} & \left(x_{1}-c\right)^{2 \alpha} & \cdots & \left(x_{1}-c\right)^{N \alpha} \\
1 & \left(x_{2}-c\right)^{\alpha} & \left(x_{2}-c\right)^{2 \alpha} & \cdots & \left(x_{2}-c\right)^{N \alpha} \\
\vdots & \vdots & \vdots & \ddots & \vdots \\
1 & \left(x_{N}-c\right)^{\alpha} & \left(x_{N}-c\right)^{2 \alpha} & \cdots & \left(x_{N}-c\right)^{N \alpha}
\end{array}\right]
$$$$
\mathbf{P}_{\mathbf{r}}=\left[\begin{array}{ccccc}
p\left(x_{0}\right) & 0 & 0 & \cdots & 0 \\
0 & p\left(x_{1}\right) & 0 & \cdots & 0 \\
0 & 0 & p\left(x_{2}\right) & \cdots & 0 \\
\vdots & \vdots & \vdots & \ddots & \vdots \\
0 & 0 & 0 & \cdots & p\left(x_{N}\right)
\end{array}\right], \mathbf{F}=\left[\begin{array}{c}
f\left(x_{0}\right) \\
f\left(x_{1}\right) \\
f\left(x_{2}\right) \\
\vdots \\
f\left(x_{N}\right)
\end{array}\right]
$$$$
\mathbf{B}_{\mathbf{q}_{r}, \mathrm{c}}=\left[\begin{array}{cccccc}
\left(q_{r}-c\right)^{0 \alpha} & 0 & 0 & 0 & \cdots & 0 \\
0 & \left(q_{r}-c\right)^{1 \alpha} & 0 & 0 & \cdots & 0 \\
0 & 0 & \left(q_{r}-c\right)^{2 \alpha} & 0 & \cdots & 0 \\
0 & 0 & 0 & \left(q_{r}-c\right)^{3 \alpha} & \cdots & 0 \\
\vdots & \vdots & \vdots & \vdots & \ddots & \vdots \\
0 & 0 & 0 & 0 & \cdots & \left(q_{r}-c\right)^{N \alpha}
\end{array}\right]
$$

Hence, the fundamental matrix equation (11) corresponding to Eq. (1) can be written in the form

$$
\mathbf{W A}=\mathbf{F} \text { or }[\mathbf{W} ; \mathbf{F}], \mathbf{W}=\left[w_{i, j}\right], i, j=0,1, \ldots, N
$$

$$
\mathbf{W}=\mathbf{X} \mathbf{M}_{i} \mathbf{M}_{0}-\sum_{r=0}^{m} \mathbf{P}_{\mathbf{r}} \mathbf{X} \mathbf{B}_{\mathbf{q}_{\mathbf{r}}, \mathbf{c}} \mathbf{M}_{\mathbf{0}}
$$

Consequently, to find the unknown fractional Taylor coefficients $D_{*}^{k \alpha} y(c), \quad k=0,1, \ldots, N$ related with the approximate solution of the problem consisting of Eq. (1) and conditions (2), by replacing the $m$ row matrices (9) by the last $m$ rows of the matrix (13), we have augmented matrix

$$
\left[\mathbf{W}^{*} ; \mathbf{F}^{*}\right]=\left[\begin{array}{cccccc}
w_{00} & w_{01} & \cdots & w_{0 N} & ; & f\left(x_{0}\right) \\
w_{10} & w_{11} & \cdots & w_{1 N} & ; & f\left(x_{1}\right) \\
\vdots & \vdots & \ddots & \vdots & ; & \vdots \\
w_{N-m 0} & w_{N-m 1} & \cdots & w_{N-m N} & ; & f\left(x_{N-m}\right) \\
u_{00} & u_{01} & \cdots & u_{0 N} & ; & \lambda_{0} \\
u_{10} & u_{11} & \cdots & u_{1 N} & ; & \lambda_{1} \\
\vdots & \vdots & \ddots & \vdots & ; & \vdots \\
u_{m-10} & u_{m-11} & \cdots & u_{m-1 N} & ; & \lambda_{m-1}
\end{array}\right]
$$

or the corresponding matrix equation

$$
\mathbf{W}^{*} \mathbf{A}=\mathbf{F}^{*}
$$

If $\operatorname{det} \mathbf{W}^{*} \neq 0$, we can write Eq.(1) as

$$
\mathbf{A}=\left(\mathbf{W}^{*}\right)^{-1} \mathbf{F}^{*}
$$

and the matrtix $\mathbf{A}$ is uniquely determined. Therefore, the approximate solution is given by the truncated fractional Taylor series

$$
y_{N}(x)=\sum_{i=0}^{N} \frac{(x-c)^{k \alpha}}{\Gamma(k \alpha+1)}\left(D_{a}^{k \alpha} y\right)(c) .
$$

\subsection{Accuracy of the solution and error bound}

To investigate the convergence, we define the error function as: [28]

$$
e_{N}(x)=y(x)-y_{N}(x)
$$

where $y(x)$ and $y_{N}(x)$ are the exact and the computed solution of the Eq.(1), respectively. Substituting $y_{N}(x)$ into Eq.(1) leads to

$$
\left(D_{*}^{i \alpha} y_{N}(x)-\sum_{r=0}^{m} p_{r}(x) y_{N}\left(q_{r} x\right)\right)=f(x)+\rho_{N}(x)
$$

where $\rho_{N}(x)$ is the perturbation term that can be obtained by substituting the computed solution $y_{N}(x)$ into Eq.(1), i.e.

$$
\rho_{N}(x)=\left(D_{*}^{i \alpha} y_{N}(x)-\sum_{r=0}^{m} p_{r}(x) y_{N}\left(q_{r} x\right)\right)-f(x)
$$

Now, by subtracting (17) from (1) and using (16), the error function $e_{N}(x)$ satisfies:

$$
-\rho_{N}(x)=\left(D_{*}^{i \alpha} e_{N}(x)-\sum_{r=0}^{m} p_{r}(x) e_{N}\left(q_{r} x\right)\right)
$$

where 
Theorem 4.1 $f$ and $p_{r}(x)$ be continuous functions on $[a, b]$. Suppose that for some positive and we have

$\left|D^{\alpha(N+1)} y(x)\right| \leq M, \forall x \in[0, b], \alpha>\beta$

Then

$$
\lim _{N \rightarrow \infty} p_{N}=0
$$

Proof: Suppose that the solution $y(x)$ and computed solution $y_{N}(x)$ of Eq.(1) are approximated by their Taylor expansions about zero. Then we may write

$e_{N}(x)=\sum_{k=N+1}^{\infty} \frac{x^{k \alpha}}{\Gamma(k+1)}\left(D^{k \alpha} y(x)\right)_{x=0}$

which can be represented as

$e_{N}(x)=\frac{x^{(N+1) \alpha}}{\Gamma(N+1)}\left(D^{(N+1) \alpha} y(\xi)\right), \xi \in(0, x)$

for some $\xi \in(0, x)$ by generalized Taylor theorem. Replacing $e_{N}(x)$ by (22) into (19) gives

$-\rho_{N}(x)=\left(D_{*}^{i \alpha} \frac{x^{(N+1) \alpha}}{\Gamma(N+1)}\left(D^{(N+1) \alpha} y(\xi)\right)-\sum_{r=0}^{m} p_{r}(x) \frac{\left(q_{r} x\right)^{(N+1) \alpha}}{\Gamma(N+1)}\left(D^{(N+1) \alpha} y(\xi)\right)\right)$

Therefore, we have

$\left|\rho_{N}(x)\right| \leq \frac{\left(D^{(N+1) \alpha} y(\xi)\right)}{\Gamma(N+1)}\left(\frac{\Gamma(N \alpha+1)}{\Gamma((N+1) \alpha+1)} b^{(N+1) \alpha}-m^{2} M\left(q^{*} b\right)^{N+1}\right)$

where $\left|p_{r}(x)\right| \leq M$ and $q^{*}=\max _{r}\left\{q_{r}\right\}$.

The proof is complete.

Theorem 4.2 Under the assumptions of Theroem 4.1, we have $\lim _{N \rightarrow \infty} e_{N}=0$.

Proof: Let assume

$$
D^{*}=\left(D_{*}^{i \alpha}-\sum_{r=0}^{m} p_{r}(x)\left(q_{r} x\right)\right)
$$

Then, the Eq.(18) can be written as

$$
D^{*} e_{N}(x)=-\rho_{N}(x)
$$

Under the assumption, $\lim _{N \rightarrow \infty} p_{N}=0$ and the Eq.(1) has unique solution [2]. Then, the operator $D^{*}$ is invertible. Hence $\lim _{N \rightarrow \infty} e_{N}=0$.

We can easily check the accuracy of the method. Since the truncated fractional Taylor series (3) is an approximate solution of Eq.(1), when the solution $y(x)$ and its fractional derivatives are substituted in Eq.(1), the resulting equation must be satisfied approximately; that is for $x=x_{q} \in[a, b], q=0,1,2, \ldots$

$$
E\left(x_{q}\right)=\left|D_{*}^{i \alpha} y(x)-f(x)-\sum_{r=0}^{m} p_{r}(x) y\left(q_{r} x\right)\right| \cong 0
$$

\section{EXAMPLES}

In this section, we give a numerical example which is presented to demonstrate the effectiveness of the proposed method.

Example 1: Consider the fractional pantograph equation

$$
D_{*}^{1 / 2} y(x)=2 y\left(\frac{3}{2} x\right)+\frac{8 x^{3 / 2}}{3 \sqrt{\pi}}-\frac{9 x^{2}}{2}
$$

with initial conditions

$$
y(0)=0, y(1)=1
$$

Here is, $P_{0}=2, q_{r}=\frac{3}{2}$. We assume that $\alpha=1 / 2,0 \leq x \leq 1$ and we seek the approximate solutions $y_{N}(x)$ by fractional Taylor polynomial, for $c=0, N=6$

$$
y_{6}(x)=\sum_{k=0}^{6} \frac{x^{k \alpha}}{\Gamma(k \alpha+1)}\left(D_{*}^{k \alpha} y(x)\right)_{x=0} .
$$

With collocation points are

$$
\begin{aligned}
& x_{0}=0, x_{1}=1 / 6, x_{2}=1 / 3, x_{3}=1 / 2, x_{4}=2 / 3, x_{5}=5 / 6 \\
& x_{6}=1
\end{aligned}
$$

Fundamental matrix relation of this problem is

$$
\left(\mathbf{X M}_{1} \mathbf{M}_{0}-\mathbf{P}_{\mathbf{0}} \mathbf{X B} \mathbf{q}_{\mathrm{r}, 0} \mathbf{M}_{\mathbf{0}}\right) \mathbf{A}=\mathbf{F}
$$

where

$$
\begin{aligned}
X & =\left[\begin{array}{ccccccc}
1 & 0 & 0 & 0 & 0 & 0 & 0 \\
1 & \sqrt{6} / 6 & 1 / 6 & \sqrt{6} / 36 & 1 / 36 & \sqrt{6} / 216 & 1 / 216 \\
1 & \sqrt{3} / 3 & 1 / 3 & \sqrt{3} / 9 & 1 / 9 & \sqrt{3} / 27 & 1 / 27 \\
1 & \sqrt{2} / 2 & 1 / 2 & \sqrt{2} / 4 & 1 / 4 & \sqrt{2} / 8 & 1 / 8 \\
1 & \sqrt{6} / 3 & 2 / 3 & 2 \sqrt{6} / 9 & 4 / 9 & 4 \sqrt{6} / 27 & 8 / 27 \\
1 & \sqrt{30} / 6 & 5 / 6 & 5 \sqrt{30} / 36 & 25 / 36 & 25 \sqrt{30} 216 & 125 / 216 \\
1 & 1 & 1 & 1 & 1 & 1 & 1
\end{array}\right] \\
\mathbf{M}_{0} & =\left[\begin{array}{ccccccc}
1 & 0 & 0 & 0 & 0 & 0 & 0 \\
0 & 2 / \sqrt{\pi} & 0 & 0 & 0 & 0 & 0 \\
0 & 0 & 1 & 0 & 0 & 0 & 0 \\
0 & 0 & 0 & 4 / 3 \sqrt{\pi} & 0 & 0 & 0 \\
0 & 0 & 0 & 0 & 1 / 2 & 0 & 0 \\
0 & 0 & 0 & 0 & 0 & 8 / 15 \sqrt{\pi} & 0 \\
0 & 0 & 0 & 0 & 0 & 0 & 1 / 6
\end{array}\right]
\end{aligned}
$$$$
\mathbf{P}_{0}=\left[\begin{array}{lllllll}
2 & 0 & 0 & 0 & 0 & 0 & 0 \\
0 & 2 & 0 & 0 & 0 & 0 & 0 \\
0 & 0 & 2 & 0 & 0 & 0 & 0 \\
0 & 0 & 0 & 2 & 0 & 0 & 0 \\
0 & 0 & 0 & 0 & 2 & 0 & 0 \\
0 & 0 & 0 & 0 & 0 & 2 & 0 \\
0 & 0 & 0 & 0 & 0 & 0 & 2
\end{array}\right]
$$ 


$$
\mathbf{B}_{q_{r}, 0}=\left[\begin{array}{ccccccc}
1 & 0 & 0 & 0 & 0 & 0 & 0 \\
0 & \sqrt{6} / 2 & 0 & 0 & 0 & 0 & 0 \\
0 & 0 & 3 / 2 & 0 & 0 & 0 & 0 \\
0 & 0 & 0 & 3 \sqrt{6} / 4 & 0 & 0 & 0 \\
0 & 0 & 0 & 0 & 9 / 4 & 0 & 0 \\
0 & 0 & 0 & 0 & 0 & 9 \sqrt{6} / 8 & 0 \\
0 & 0 & 0 & 0 & 0 & 0 & 27 / 8
\end{array}\right]
$$$$
\mathbf{F}=\left[\begin{array}{c}
0 \\
-0.022631 \\
-0.210457 \\
-0.593076 \\
-1.181050 \\
-1.980453 \\
-2.995494
\end{array}\right]
$$

Also, we have the matrix representation of conditions as,

$$
\begin{aligned}
& y(0)=\left[\begin{array}{lllllll}
1 & 0 & 0 & 0 & 0 & 0 & 0
\end{array}\right] \mathbf{A}=[0] \\
& y(1)=\left[\begin{array}{lllllll}
1 & 2 / \sqrt{\pi} & 1 & 4 / 3 \sqrt{\pi} & 1 / 2 & 8 / 15 \sqrt{\pi} & 1 / 6
\end{array}\right] \mathbf{A}=[1]
\end{aligned}
$$

Therefore, we can calculate the new augmented matrix based on conditions and solving this systems we obtain for $N=6$

$\mathbf{A}=\left[\begin{array}{lllllll}0 & 0.199364 e-5 & -0.333911 e-5 & 0.242222 e-5 & 1.999975 & 0.187881 e-5 & 0.5209188 e-5\end{array}\right]$ Finally, we substitute the elements of the fractional Taylor coefficient matrix $\mathbf{A}$ into Eq.(3). Thus the approximate solution of equation becomes

$$
y_{6}=0.18 e-12 x-0,589 e-12 x^{3 / 2}+0.99 x^{2}+0.18 e-12 x^{5 / 2}+0.35 e-13 x^{3}
$$

Which is an appropriate solution. The exact solution of Example 1 is $y(x)=x^{2}$.

Comparison of numerical results with the exact solution is shown in Table 1 and plotted the numerical results in Fig.1 for various $N$. Figure 1 shows that the exact solution and approximate solutions is overlapping Figure 2 shows that the errors result for various $\mathrm{N}$

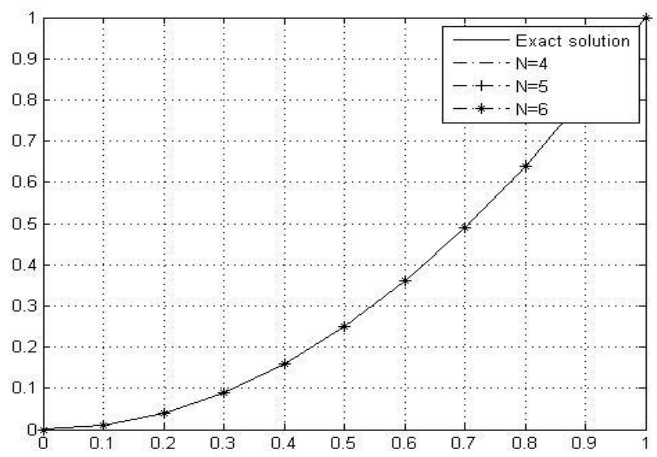

Fig1:The numerical results of Example1 for various $\mathbf{N}$

\begin{tabular}{|c|c|c|c|c|c|c|c|}
\hline $\mathbf{x}$ & $\begin{array}{c}\text { Exact } \\
\text { Solution }\end{array}$ & $N=4$ & $N_{e}=4$ & $N=5$ & $N_{e}=5$ & $N=6$ & $N_{e}=6$ \\
\hline 0.0 & 0.000000 & 0.000000 & $0.000 \mathrm{E}-0$ & 0.000000 & $0.000 \mathrm{E}-0$ & 0.000000 & $0.000 \mathrm{E}-0$ \\
\hline 0.1 & 0.010000 & 0.009999 & $0.314 \mathrm{E}-9$ & 0.010000 & $0.31 \mathrm{E}-11$ & 0.009999 & $0.45 \mathrm{E}-14$ \\
\hline 0.2 & 0.040000 & 0.039999 & $0.225 E-9$ & 0.040000 & $0.75 \mathrm{E}-11$ & 0.039999 & $0.31 \mathrm{E}-14$ \\
\hline 0.3 & 0.090000 & 0.090000 & $0.115 \mathrm{E}-7$ & 0.090000 & $0.95 \mathrm{E}-11$ & 0.089999 & $0.53 E-15$ \\
\hline 0.4 & 0.160000 & 0.160003 & $0.25 \mathrm{E}-10$ & 0.160000 & $0.88 \mathrm{E}-11$ & 0.160001 & $0.13 E-14$ \\
\hline 0.5 & 0.250000 & 0.25000 & $0.330 \mathrm{E}-9$ & 0.250000 & $0.66 \mathrm{E}-11$ & 0.250004 & $0.35 \mathrm{E}-14$ \\
\hline 0.6 & 0.360000 & 0.360008 & $0.561 E-9$ & 0.360000 & $0.33 \mathrm{E}-11$ & 0.360005 & $0.53 E-14$ \\
\hline 0.7 & 0.490000 & 0.490008 & $0.754 \mathrm{E}-9$ & 0.490000 & $0.66 \mathrm{E}-12$ & 0.490006 & $0.55 \mathrm{E}-14$ \\
\hline 0.8 & 0.640000 & 0.640007 & $0.779 E-9$ & 0.639999 & $0.12 \mathrm{E}-11$ & 0.640004 & $0.38 \mathrm{E}-14$ \\
\hline 0.9 & 0.810000 & 0.810005 & $0.710 \mathrm{E}-9$ & 0.809999 & $0.15 \mathrm{E}-11$ & 0.810002 & $0.22 \mathrm{E}-14$ \\
\hline 1.0 & 1.000000 & 1.000000 & $0.114 \mathrm{E}-9$ & 1.000000 & $0.82 \mathrm{E}-12$ & 0.999990 & $0.15 \mathrm{E}-14$ \\
\hline
\end{tabular}

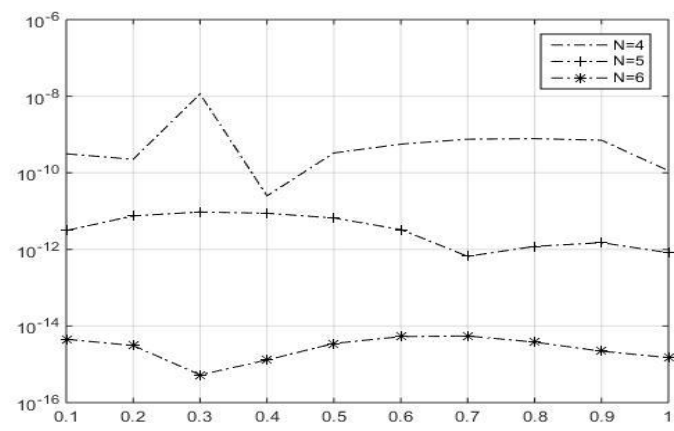

Fig. 2: The numerical results of erros for various $N$

Table 1: Numerical result for Example 1 
Example 2: Consider the following fractional Pantograph equation

$$
D_{*}^{2} y(x)+D_{*}^{3 / 2} y(x)+y(x)=y\left(\frac{1}{2} x\right)+f(x)
$$

with initial conditions

$$
y(0)=0, y(1)=1 .
$$

Where $f(x)=x^{2}+4 \sqrt{\frac{x}{\pi}}+2-\frac{x^{2}}{4}$.

Then, $P_{0}=1, q_{r}=\frac{1}{2}$. We assume that $\alpha=1 / 2,0 \leq x \leq 1$ and we seek the approximate solutions $y_{N}(x)$ by fractional Taylor polynomial, for $c=0, N=6$

$$
y_{6}(x)=\sum_{k=0}^{6} \frac{x^{k \alpha}}{\Gamma(k \alpha+1)}\left(D_{*}^{k \alpha} y(x)\right)_{x=0} .
$$

With collocation points are

$x_{0}=0, x_{1}=1 / 6, x_{2}=1 / 3, x_{3}=1 / 2, x_{4}=2 / 3, x_{5}=5 / 6$

$x_{6}=1$

Fundamental matrix relation of this problem is

$\left(\mathbf{X M}_{4} \mathbf{M}_{0}+\mathbf{X M}_{3} \mathbf{M}_{0}+\mathbf{X M M} \mathbf{M}_{0}-\mathbf{P}_{\mathbf{0}} \mathbf{X B} \mathbf{q}_{\mathrm{r}, 0} \mathbf{M}_{\mathbf{0}}\right) \mathbf{A}=\mathbf{F}$

Where

$$
\mathbf{X}=\left[\begin{array}{ccccccc}
1 & 0 & 0 & 0 & 0 & 0 & 0 \\
1 & \sqrt{6} / 6 & 1 / 6 & \sqrt{6} / 36 & 1 / 36 & \sqrt{6} / 216 & 1 / 216 \\
1 & \sqrt{3} / 3 & 1 / 3 & \sqrt{3} / 9 & 1 / 9 & \sqrt{3} / 27 & 1 / 27 \\
1 & \sqrt{2} / 2 & 1 / 2 & \sqrt{2} / 4 & 1 / 4 & \sqrt{2} / 8 & 1 / 8 \\
1 & \sqrt{6} / 3 & 2 / 3 & 2 \sqrt{6} / 9 & 4 / 9 & 4 \sqrt{6} / 27 & 8 / 27 \\
1 & \sqrt{30} / 6 & 5 / 6 & 5 \sqrt{30} / 36 & 25 / 36 & 25 \sqrt{30} 216 & 125 / 216 \\
1 & 1 & 1 & 1 & 1 & 1 & 1
\end{array}\right]
$$$$
\mathbf{M}_{3}=\left[\begin{array}{ccccccc}
0 & 0 & 0 & 1 & 0 & 0 & 0 \\
0 & 0 & 0 & 0 & 2 / \sqrt{\pi} & 0 & 0 \\
0 & 0 & 0 & 0 & 0 & 1 & 0 \\
0 & 0 & 0 & 0 & 0 & 0 & 4 / 3 \sqrt{\pi} \\
0 & 0 & 0 & 0 & 0 & 0 & 0 \\
0 & 0 & 0 & 0 & 0 & 0 & 0 \\
0 & 0 & 0 & 0 & 0 & 0 & 0
\end{array}\right] \mathbf{F}=\left[\begin{array}{c}
2 \\
2.942151 \\
3.386273 \\
3.783269 \\
4.175968 \\
4.580962 \\
5.006758
\end{array}\right]
$$$$
\mathbf{P}_{0}=\left[\begin{array}{lllllll}
1 & 0 & 0 & 0 & 0 & 0 & 0 \\
0 & 1 & 0 & 0 & 0 & 0 & 0 \\
0 & 0 & 1 & 0 & 0 & 0 & 0 \\
0 & 0 & 0 & 1 & 0 & 0 & 0 \\
0 & 0 & 0 & 0 & 1 & 0 & 0 \\
0 & 0 & 0 & 0 & 0 & 1 & 0 \\
0 & 0 & 0 & 0 & 0 & 0 & 1
\end{array}\right] \mathbf{B}_{q, 0}=\left[\begin{array}{ccccccc}
1 & 0 & 0 & 0 & 0 & 0 & 0 \\
0 & \sqrt{2} / 2 & 0 & 0 & 0 & 0 & 0 \\
0 & 0 & 1 / 2 & 0 & 0 & 0 & 0 \\
0 & 0 & 0 & \sqrt{2} / 4 & 0 & 0 & 0 \\
0 & 0 & 0 & 0 & 1 / 4 & 0 & 0 \\
0 & 0 & 0 & 0 & 0 & \sqrt{2} / 8 & 0 \\
0 & 0 & 0 & 0 & 0 & 0 & 1 / 8
\end{array}\right]
$$

Also, we have the matrix representation of conditions as, $y(0)=\left[\begin{array}{lllllll}1 & 0 & 0 & 0 & 0 & 0 & 0\end{array}\right] \mathbf{A}=[0]$

$y(1)=\left[\begin{array}{lllllll}1 & 2 / \sqrt{\pi} & 1 & 4 / 3 \sqrt{\pi} & 1 / 2 & 8 / 15 \sqrt{\pi} & 1 / 6\end{array}\right] \mathrm{A}=[1]$ and so we solve the equation and obtain the coefficients of the fractional Taylor polynomials

$\mathbf{A}=\left[\begin{array}{lllllll}0 & 0 & -0.181959 e-12 & 0.783892 e-12 & 1.999999 & 0.301528 e-12 & 0.210585 e-12\end{array}\right]$ Hence, for $N=6$, the approximate solution of Example 2 is given $_{y_{6}}=0.18 e-12 x-0,58 e-12 x^{3 / 2}+0.99 x^{2}+0.90 e-13 x^{5 / 2}+0.35 e-13 x^{3}$

The exact solution of Example 2 is $y(x)=x^{2}$.

Comparison of numerical results with the exact solution is shown in Table 2 and plotted the numerical results in Fig. 3 for various $N$. Figure 4 shows that the exact solution and approximate solutions is overlapping, Figure 4 shows that the errors result for various $\mathrm{N}$

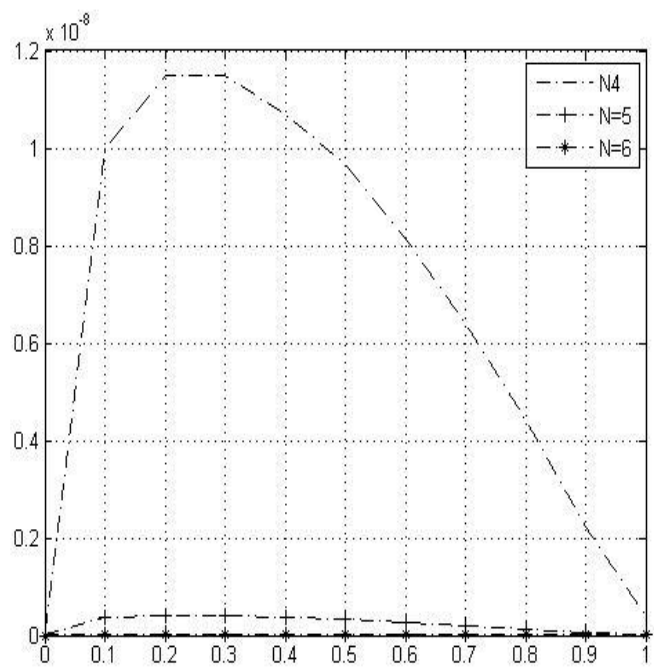

Fig. 3: The numerical results of errors for various $N$

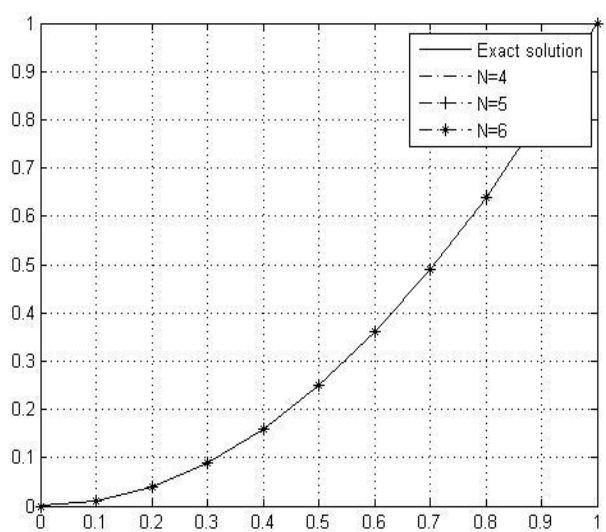

Fig. 4: The numerical results of Example2 for various $\mathbf{N}$

Table 2: Numerical result for Example 2

\begin{tabular}{|c|c|c|c|c|c|c|c|}
\hline $\mathbf{x}$ & $\begin{array}{c}\text { Exact } \\
\text { Solution }\end{array}$ & $\mathbf{N}=\mathbf{4}$ & $\mathbf{N}_{\mathrm{e}}=\mathbf{4}$ & $\mathbf{N}=\mathbf{5}$ & $\mathbf{N}_{\mathrm{e}}=\mathbf{5}$ & $\mathbf{N}=\mathbf{6}$ & $\mathbf{N}_{\mathrm{e}}=\mathbf{6}$ \\
\hline 0.0 & 0.000000 & 0.000000 & $0.000 \mathrm{E}-0$ & 0.000000 & $0.000 \mathrm{E}-0$ & 0.000000 & $0.000 \mathrm{E}-0$ \\
\hline
\end{tabular}




\begin{tabular}{|c|c|c|c|c|c|c|c|}
\hline 0.1 & 0.010000 & 0.009998 & $0.100 \mathrm{E}-7$ & 0.009999 & $0.380 \mathrm{E}-9$ & 0.009999 & $0.22 \mathrm{E}-10$ \\
\hline 0.2 & 0.040000 & 0.039999 & $0.115 \mathrm{E}-7$ & 0.039999 & $0.421-\mathrm{E} 9$ & 0.039999 & $0.24 \mathrm{E}-10$ \\
\hline 0.3 & 0.090000 & 0.089999 & $0.115 \mathrm{E}-7$ & 0.089999 & $0.411 \mathrm{E}-9$ & 0.089999 & $0.23 \mathrm{E}-10$ \\
\hline 0.4 & 0.160000 & 0.159999 & $0.107 \mathrm{E}-7$ & 0.159999 & $0.377 \mathrm{E}-9$ & 0.159999 & $0.21 \mathrm{E}-10$ \\
\hline 0.5 & 0.250000 & 0.249999 & $0.967 \mathrm{E}-8$ & 0.249999 & $0.329 \mathrm{E}-9$ & 0.249999 & $0.18 \mathrm{E}-10$ \\
\hline 0.6 & 0.360000 & 0.359999 & $0.811 \mathrm{E}-8$ & 0.359999 & $0.271 \mathrm{E}-9$ & 0.359999 & $0.15 \mathrm{E}-10$ \\
\hline 0.7 & 0.490000 & 0.489999 & $0.641 \mathrm{E}-8$ & 0.489999 & $0.208 \mathrm{E}-9$ & 0.489999 & $0.11 \mathrm{E}-10$ \\
\hline 0.8 & 0.640000 & 0.639999 & $0.440 \mathrm{E}-8$ & 0.639999 & $0.139 \mathrm{E}-9$ & 0.639999 & $0.77 \mathrm{E}-11$ \\
\hline 0.9 & 0.810000 & 0.809999 & $0.223 \mathrm{E}-8$ & 0.809999 & $0.67 \mathrm{E}-10$ & 0.809999 & $0.39 \mathrm{E}-11$ \\
\hline 1.0 & 1.000000 & 1.000000 & $0.372 \mathrm{E}-9$ & 1.000000 & $0.40 \mathrm{E}-11$ & 0.999999 & $0.60 \mathrm{E}-14$ \\
\hline
\end{tabular}

\section{CONCLUSION}

In this study, we present a Taylor collocation method for the numerical solutions of fractional Pantograph equation. This method transforms the fractional Pantograph equation into matrix equations. The desired approximate solutions can be determined by solving the resulting system, which can be effectively computed using symbolic computing codes on Maple 14. Example shows that Taylor collocation method has been successfully applied to finding the approximate solutions fractional Pantograph equation. Also the method can be expanded to solve fractional multi pantograph equation, fractional integro multi pantograph equation and fractional system of pantograph equation.

\section{REFERENCES}

[1] R. Hilfer (Ed.), Applications of Fractional Calculus in Physics, Academic Press, Orlando, 1999.

[2] I. Podlubny, Fractional Differential Equations, Academic Press, NewYork, 1999.

[3] A.M. Spasic, M.P. Lazarevic, Electroviscoelasticity of liquid/liquid interfaces: fractional-order model, J. Colloid Interface Sci. 282 (2005) 223-230.

[4] Muroya, Y., E. Ishiwata and H. Brunner, 2003. On the attainable order of collocation methods for pantograph integro-differential equations. J. Comput. Appl. Math., 152: $347-366$

[5] Shadia, M., 1992. Numerical Solution of Delay Differential and Neutral Differential Equations Using Spline Methods. Ph.D Thesis, Assuit University

[6] Li, D. and M.Z. Liu, 2005. Runge-Kutta methods for the multi-pantograph delay equation. Appl. Math. Comput., 163: 383-395.

[7] Evans, D.J. and K.R. Raslan, 2005. The Adomian decomposition method for solving delay differential equation. Int. J. Comput. Math., 82 (1): 49-54

[8] E. Yusufoglu, An efficient algorithm for solving generalized pantograph equations with linear functional argument, App. Math. Comp., 217 (2010) 3591-3595
[9] Sezer M, Yalcinbas S, Sahin N. Approximate solution of multi-pantograph equation with variable coeffi-cients. J Comput Appl Math, 2008, 214: 406-416

[10] $\mathrm{Yu} \mathrm{Z} \mathrm{H.} \mathrm{Variational} \mathrm{iteration} \mathrm{method} \mathrm{for} \mathrm{solving} \mathrm{the}$ multi-pantograph delay equation. Phys Lett A, 2008,372: 6475-6479

[11] Liu M Z, Li D. Properties of analytic solution and numerical solution of multi-pantograph equation. Appl Math Comput, 2004, 155: 853-871

[12] Z. Fan, M. Liu, W. Cao, Existence and uniqueness of the solutions and convergence of semi-implicit Euler methods for stochastic pantograph equations, J. Math. Anal. Appl. 325 (2007) 1142-1159.

[13] S.Momani,Z.Odibat, Numerical comparison of methods for solving linear differential equations of fractional order, Chaos, Solitons and Fractals 31 (2007) 12481255 .

[14] S.S. Ray, R.K. Bera, Solution of an extraordinary dfferential equation by Adomian decomposition method, J. Appl. Math. 4 (2004) 331-338.

[15] A.M.A. El-Sayed, I.L. El-Kalla, E.A.A. Ziada, Analytical and numerical solutions of multi-term nonlinear fractional orders differential equations, Appl. Numer. Math. 60 (2010) 788-797.

[16] O. Abdulaziz, I. Hashim, S. Momani, Application of homotopy-perturbation method to fractional IVPs, J. Comput. Appl. Math. 216 (2008) 574-584.

[17] S. Yanga, A. Xiao, H. Su, Convergence of the variational iteration method for solving multi-order fractional differential equations, Comput. Math. Appl. 60 (2010) 2871-2879.

[18] Z. Odibat, S. Momani, H. Xu, A reliable algorithm of homotopy analysis method for solving nonlinear fractional differential equations, Appl. Math.Model. 34 (2010) 593-600.

[19] E.H. Doha, A.H. Bhrawy, S.S. Ezz-Eldien, A Chebyshev spectral method based on operational matrix for initial 
and boundary value problems of fractional order, Computers and Mathematics with Applications 62 (2011) 2364-2373.

[20] M. Gülsu, M. Sezer, A Taylor polynomial approach for solving differential difference equations, J. Comp. Appl. Math. 186 (2006) 349-364

[21] M. Sezer, Taylor polynomial solution of Volterra Integral equations, Internat. J. Math. Ed. Sci. Technol. 25 (1994) 625-633.

[22] A. Karamete, M. Sezer, A Taylor collocation method for the solution of linear integrodifferential equations, Int. J. Comput. Math. 79 (2002) 987-1000.

[23] M. Gulsu, M. Sezer, Approximations to the solution of linear Fredholm integro-differential-difference equation of high order, J. Franklin Inst. 343 (2006) 720-737.

[24] M. Sezer, M. Gulsu, Polynomial solution of the most general linear Fredholm-Volterra integro differential- difference equations by means of Taylor ollocation method, Appl. Math. Comput. 185 (2007) 646-657.

[25] M. Gülsu, Y. Öztürk, M. Sezer, A new collocation method for solution of mixed linear integro differential difference equations, Appl. Math. Comp. 216 (2010) 2183-2198.

[26] Z. Odibat, N.T. Shawagfeh, Generalized Taylor's Formula. Appl. Math.Comput. 186 (2007) 286-293.

[27] Y. Keskin, O. Karaoğlu, S. servi, G. Oturaç, The approximate solution of high order linear fractional 52ifferential equations with variable coefficients in terms of generalized Taylor polynomials, Math. Comp. Appl. 16 (2011) 617-629.

[28] Saeedi L., Tari A., Masuleh Momami H.S. 2013. Nuerical Solution of Some Nonlinear Volterra Integral Equations of First Kind, App. App. Math. 1932-9466. 